\title{
Effect of wall properties on the peristaltic flow of a non-Newtonian fluid
}

\author{
Maryiam Javed $^{\mathrm{a}, *}$, T. Hayat ${ }^{\mathrm{b}, \mathrm{c}}$ and A. Alsaedi ${ }^{\mathrm{c}}$ \\ ${ }^{a}$ Department of Applied Mathematics and Statistics, Institute of Space Technology, Islamabad, Pakistan \\ ${ }^{\mathrm{b}}$ Department of Mathematics, Quaid-i-Azam University, Islamabad, Pakistan \\ ${ }^{\mathrm{c}}$ NAAM Research Group, Department of Mathematics, King Abdulaziz University, Jeddah, Saudi Arabia
}

\begin{abstract}
The effect of wall properties is analyzed on the peristaltic motion of a Burgers' fluid. Sinusoidal waves are considered on the channel walls. Small amplitude assumption is taken into account to solve the required boundary value problem. Various flow parameters of interest are sketched and discussed through graphs. Comparative study of the mean velocity for different fluid models is presented the mean velocity is found maximum in viscous fluid when compared with the Maxwell, Oldroyd-B and Burgers' fluids.
\end{abstract}

Keywords: Burgers' fluid, peristaltic flow, compliant wall

\section{Introduction}

Recently considerable attention has been focused to the peristaltic flows through tubes/channels. This type of flows are significant in both theoretical and industrial contexts. Such flows have vital role in the bio-fluid mechanics and specifically these may be involved in urine transport from kidney to bladder, swallowing foods through oesophagus, chyme movement in small intestines and many others. Several theoretical and experimental studies have been undertaken to understand peristalsis through abrupt changes in geometry and realistic assumptions and its complete survey in the domain of biomechanics has been given by Jaffrin and Shapiro [1]. It is an established fact now that many of the physiological fluids are non-Newtonian. In view of this reason many investigators have concentrated recently on the hydrodynamic and magnetohydrodynamic (MHD) peristaltic flows of Newtonian/non-Newtonian fluids.

\footnotetext{
*Corresponding author: Maryiam Javed, Department of Applied Mathematics and Statistics, Institute of Space Technology 2750, Islamabad 44000, Pakistan. Tel.: +92 51 9075585; Fax: +92 51 9273310; E-mail: maina786@hotmail.com.
}

Many recent theoretical investigations [2-16] have been contributed by the researchers using analytical and numerical methods.

All the above mentioned studies do not discuss the mechanism of peristaltic transport with compliant walls of channels. Haroun [17] discussed peristaltic flows of viscous fluids in a two-dimensional channel with compliant walls. He presented the flow analysis under the assumption of small amplitude ratio. Mekheimer and Wahab [18] analyzed the peristaltic flow of a compressible fluid induced by a surface acoustic wave in a microchannel. Mustafa et al. [19] studied the influence of wall properties on the peristaltic flow of a nanofluid. Hayat et al. [20] analyzed the effect of wall compliance on flow of compressible nonNewtonian fluid. Kumari and Radhakrishnamacharya [21] discussed the slip and magnetic field effects on peristaltic flow in an inclined channel with wall properties. The main purpose of the present investigation is to put forward the peristaltic flow analysis of nonNewtonian fluids in a compliant walls channel. For that constitutive equations of a subclass of rate type fluids namely Burgers' model have been used. The study of analytic solutions for such fluids is significantly 
more difficult and is currently in its infaney. The paper is organized as follows. In Section 2, we provide the mathematical modelling and resulting problem statement. The nonlinear differential equations with appropriate boundary conditions have been solved analytically in Section 3. Section 4 describes the graphical results. Final remarks are summarized in Section 5.

\section{Problem statement}

We consider an incompressible homogenous Burgers' fluid in a channel with uniform width $2 h$. The flow is engendered by a small amplitude sinusoidal waves on the compliant walls of the channel. The geometry of the walls is

$$
y= \pm h \pm \eta
$$

where the vertical displacement $\eta$ of the upper wall from its normal position is given by

$$
\eta=a \cos \frac{2 \pi}{\lambda}(x-c t) .
$$

In above equation $a$ denotes the wave amplitude, $c$ the constant wave speed, $\bar{\lambda}$ the wavelength and for lower wall $\eta$ is replaced by $-\eta$. We choose $x$-axis in the direction of wave propagation and $y$ normal to the $x$-axis.

Expression of compliant wall is written as

$$
\left[m \frac{\partial^{2}}{\partial t^{2}}+d \frac{\partial}{\partial t}+B \frac{\partial^{4}}{\partial x^{4}}-T \frac{\partial^{2}}{\partial x^{2}}+K\right] \eta=p-p_{0}
$$

in which $m$ indicates the plate mass per unit area, $d$ the wall damping coefficient, $B$ the flexural rigidity of the plate, $T$ the longitudinal tension per unit width, $K$ the spring stiffness and $p_{0}$ the pressure on the outside surface of the wall. Let us suppose $p_{0}=0$ and the channel walls inextensible so that only their lateral motions normal to the undeformed positions occur. The horizontal displacement of the walls is taken zero. The subjected boundary conditions are

$\Psi_{y}=0 \quad$ and $\quad \Psi_{x}=\mp \frac{\partial \eta}{\partial t} \quad$ at $y= \pm h \pm \eta$,

where the stream function $\Psi(x, y, t)$ is defined by the expressions given below

$$
u=\frac{\partial \Psi}{\partial y}, \quad v=-\frac{\partial \Psi}{\partial x} .
$$

Here $u$ and $v$ are the $x$ - and $y$-components of the velocity $\mathbf{V}$ respectively.

In absence of body forces, the equations which can govern the flow of an incompressible fluid are

$$
\begin{aligned}
& \operatorname{div} \mathbf{V}=0 \\
& \rho \frac{d \mathbf{V}}{d t}=-\nabla p+\operatorname{div} \mathbf{S},
\end{aligned}
$$

where $p$ is the pressure, and an extra stress tensor $\mathbf{S}$ in a Burgers' fluid has the following expression

$$
\mathbf{S}+\lambda \frac{D \mathbf{S}}{D t}+\beta \frac{D^{2} \mathbf{S}}{D t^{2}}=\mu\left(1+\lambda_{r} \frac{D}{D t}\right) \mathbf{A}_{1}
$$

in which $\mu$ is the dynamic viscosity, $\mathbf{A}_{1}$ the first RivlinErickson tensor, $\lambda$ and $\beta$ the relaxation times, $\lambda_{r}(<\lambda)$ the retardation time and $D / D t$ the upper convected time derivative. Note that the model represented by (8) includes as the special cases of an Oldroyd-B model (for $\beta=0$ ), a Maxwell model (for $\beta=\lambda_{r}=0$ ), a second grade fluid (for $\beta=\lambda=0$ ) and a viscous fluid model (for $\beta=\lambda=\lambda_{r}=0$ ).

We note that Equation (6) is satisfied by the velocity components in Equation (5). Moreover, Equations (7) and (8) for two-dimensional flow yield the following expressions

$$
\begin{aligned}
& \rho\left(\frac{\partial u}{\partial t}+u \frac{\partial u}{\partial x}+v \frac{\partial u}{\partial y}\right)=-\frac{\partial p}{\partial x}+\frac{\partial S_{x x}}{\partial x}+\frac{\partial S_{x y}}{\partial y}, \\
& \rho\left(\frac{\partial v}{\partial t}+u \frac{\partial v}{\partial x}+v \frac{\partial v}{\partial y}\right)=-\frac{\partial p}{\partial y}+\frac{\partial S_{x y}}{\partial x}+\frac{\partial S_{y y}}{\partial y},(10 \\
& S_{x x}+\lambda\left[\left(\frac{\partial}{\partial t}+u \frac{\partial}{\partial x}+v \frac{\partial}{\partial y}\right) S_{x x}-2 \frac{\partial u}{\partial x} S_{x x}\right. \\
& \left.-2 \frac{\partial u}{\partial y} S_{x y}\right]+\beta\left\{\left[\frac{\partial^{2}}{\partial t^{2}}+\frac{\partial}{\partial t}\left(u \frac{\partial}{\partial x}+v \frac{\partial}{\partial y}\right)\right.\right. \\
& -2 \frac{\partial^{2} u}{\partial t \partial x}-4 \frac{\partial u}{\partial x} \frac{\partial}{\partial t}+\left(u \frac{\partial}{\partial x}+v \frac{\partial}{\partial y}\right) \frac{\partial}{\partial t} \\
& +\left(u \frac{\partial}{\partial x}+v \frac{\partial}{\partial y}\right)\left(u \frac{\partial}{\partial x}+v \frac{\partial}{\partial y}\right)+4\left(\frac{\partial u}{\partial x}\right)^{2} \\
& -2\left(u \frac{\partial^{2} u}{\partial x^{2}}+v \frac{\partial^{2} u}{\partial x \partial y}\right)+2 \frac{\partial u}{\partial y} \frac{\partial v}{\partial x}-4 \frac{\partial u}{\partial x} \\
& \left.\left(u \frac{\partial}{\partial x}+v \frac{\partial}{\partial y}\right)\right] S_{x x}+\left[-2 \frac{\partial^{2} u}{\partial y \partial t}-4 \frac{\partial u}{\partial y} \frac{\partial}{\partial t}\right.
\end{aligned}
$$




$$
\begin{aligned}
& +4 \frac{\partial u}{\partial x} \frac{\partial u}{\partial y}-2\left(u \frac{\partial^{2} u}{\partial x \partial y}+v \frac{\partial^{2} u}{\partial y^{2}}\right) \\
& \left.\left.-4 \frac{\partial u}{\partial y}\left(u \frac{\partial}{\partial x}+v \frac{\partial}{\partial y}\right)\right] S_{x y}+4\left(\frac{\partial u}{\partial y}\right)^{2} S_{y y}\right\} \\
= & 2 \mu \frac{\partial u}{\partial x}+2 \mu \lambda r\left[\left(\frac{\partial}{\partial t}+u \frac{\partial}{\partial x}+v \frac{\partial}{\partial y}\right) \frac{\partial u}{\partial x}\right. \\
& \left.-2\left(\frac{\partial u}{\partial x}\right)^{2}-\frac{\partial u}{\partial y}\left(\frac{\partial u}{\partial y}+\frac{\partial v}{\partial x}\right)\right] \\
S_{x y} & +\lambda\left[\left(\frac{\partial}{\partial t}+u \frac{\partial}{\partial x}+v \frac{\partial}{\partial y}\right) S_{x y}-\frac{\partial v}{\partial x} S_{x x}-\frac{\partial u}{\partial y} S_{y y}\right] \\
+ & \left\{\left[\frac{\partial^{2}}{\partial t^{2}}+\frac{\partial}{\partial t}\left(u \frac{\partial}{\partial x}+v \frac{\partial}{\partial y}\right)+\left(u \frac{\partial}{\partial x}+v \frac{\partial}{\partial y}\right) \frac{\partial}{\partial t}\right.\right. \\
+ & \left.\left(u \frac{\partial}{\partial x}+v \frac{\partial}{\partial y}\right)\left(u \frac{\partial}{\partial x}+v \frac{\partial}{\partial y}\right)+4 \frac{\partial u}{\partial y} \frac{\partial v}{\partial x}\right] S_{x y} \\
+ & {\left.\left[\frac{\partial u}{\partial y}\left(u \frac{\partial}{\partial x}+v \frac{\partial}{\partial y}\right)+2 \frac{\partial u}{\partial y} \frac{\partial v}{\partial y}\right] S_{y y}\right\} } \\
+ & {\left[\frac{\partial v}{\partial x}\left(u \frac{\partial}{\partial x}+v \frac{\partial}{\partial y}\right)+2 \frac{\partial u}{\partial x} \frac{\partial v}{\partial x}\right] S_{x x} } \\
+ & {\left[u \frac{\partial^{2} v}{\partial x^{2}}+v \frac{\partial^{2} v}{\partial x \partial y}\right)-2 \frac{\partial}{\partial x} \frac{\partial}{\partial t} }
\end{aligned}
$$

$S_{y y}+\lambda\left[\left(\frac{\partial}{\partial t}+u \frac{\partial}{\partial x}+v \frac{\partial}{\partial y}\right) S_{y y}-2 \frac{\partial v}{\partial x} S_{y x}-2 \frac{\partial v}{\partial y} S_{y y}\right]$ $+\beta\left\{\left[\frac{\partial^{2}}{\partial t^{2}}+\frac{\partial}{\partial t}\left(u \frac{\partial}{\partial x}+v \frac{\partial}{\partial y}\right)-2 \frac{\partial^{2} v}{\partial y \partial t}-4 \frac{\partial v}{\partial y} \frac{\partial}{\partial t}\right.\right.$$$
+\left(u \frac{\partial}{\partial x}+v \frac{\partial}{\partial y}\right) \frac{\partial}{\partial t}+\left(u \frac{\partial}{\partial x}+v \frac{\partial}{\partial y}\right)\left(u \frac{\partial}{\partial x}+v \frac{\partial}{\partial y}\right)
$$$$
+4\left(\frac{\partial v}{\partial y}\right)^{2}-2\left(u \frac{\partial^{2} u}{\partial x^{2}}+v \frac{\partial^{2} u}{\partial x \partial y}\right)+2 \frac{\partial u}{\partial y} \frac{\partial v}{\partial x}
$$$$
\left.-4 \frac{\partial v}{\partial y}\left(u \frac{\partial}{\partial x}+v \frac{\partial}{\partial y}\right)\right] S_{y y}+\left[-2 \frac{\partial^{2} v}{\partial x \partial t}-4 \frac{\partial v}{\partial x} \frac{\partial}{\partial t}\right.
$$$$
+4 \frac{\partial v}{\partial x} \frac{\partial v}{\partial y}-2\left(u \frac{\partial^{2} v}{\partial x^{2}}+v \frac{\partial^{2} v}{\partial x \partial y}\right)
$$

$$
\begin{aligned}
& \left.\left.-4 \frac{\partial v}{\partial x}\left(u \frac{\partial}{\partial x}+v \frac{\partial}{\partial y}\right)\right] S_{x y}+2\left(\frac{\partial v}{\partial x}\right)^{2} S_{x x}\right\} \\
& =2 \mu \frac{\partial v}{\partial y}+2 \mu \lambda_{r}\left[\left(\frac{\partial}{\partial t}+u \frac{\partial}{\partial x}\right.\right. \\
& \left.\left.+v \frac{\partial}{\partial y}\right) \frac{\partial v}{\partial y}-2\left(\frac{\partial v}{\partial y}\right)^{2}-\frac{\partial v}{\partial x}\left(\frac{\partial v}{\partial x}+\frac{\partial u}{\partial y}\right)\right] .
\end{aligned}
$$

The completion of problem formulation requires the continuity of stresses at the interfaces of the walls and the fluid, $p$ must be the same as that which acts on the fluid at $y= \pm h \pm \eta$. By Equation (9) one can write

$$
\begin{aligned}
& \frac{\partial}{\partial x}\left[m \frac{\partial^{2} \eta}{\partial t^{2}}+d \frac{\partial \eta}{\partial t}+B \frac{\partial^{4} \eta}{\partial x^{4}}-T \frac{\partial^{2} \eta}{\partial x^{2}}+K \eta\right] \\
& =-\rho\left(\Psi_{y t}+\Psi_{y} \Psi_{y x}-\Psi_{x} \Psi_{y y}\right)+S_{x x, x}+S_{x y, y}
\end{aligned}
$$

Note that the subscripts denote partial differentiation. In order to simplify the problem, we define

$$
\begin{aligned}
& \hat{x}=\frac{x}{h}, \hat{y}=\frac{y}{h}, \hat{u}=\frac{u}{c}, \hat{v}=\frac{v}{c}, \hat{t}=\frac{c t}{h}, \\
& \hat{p}=\frac{p}{\rho c^{2}}, \hat{\eta}=\frac{\eta}{h}, \hat{\Psi}=\frac{\Psi}{c h}, \\
& \hat{m}=\frac{m}{\rho h}, \hat{d}=\frac{d h}{\rho \nu}, \hat{B}=\frac{B}{\rho h v^{2}}, \\
& \hat{T}=\frac{T h}{\rho v^{2}}, \hat{K}=\frac{K h^{3}}{\rho v^{2}}, \hat{S}_{x x}=\frac{h S_{x x}}{\mu c}, \\
& \hat{S}_{x y}=\frac{h S_{x y}}{\mu c}, \hat{S}_{y y}=\frac{h S_{y y}}{\mu c}, \\
& \hat{\lambda}=\frac{c}{h} \lambda, \hat{\lambda}_{r}=\frac{c}{h} \lambda \lambda_{r} .
\end{aligned}
$$

Invoking above variables, Equations (9)-(14) after omitting hats give

$$
\begin{aligned}
& \frac{\partial}{\partial t} \nabla^{2} \Psi+\Psi_{y} \nabla^{2} \Psi_{x}-\Psi_{x} \nabla^{2} \Psi_{y} \\
& =\frac{1}{R}\left(S_{x x, x y}+S_{x y, y y}-S_{x y, x x}-S_{y y, x y}\right), \\
& S_{x x}+\lambda\left[\left(\frac{\partial}{\partial t}+\Psi_{y} \frac{\partial}{\partial x}-\Psi_{x} \frac{\partial}{\partial y}\right) S_{x x}-2 \Psi_{x y} S_{x x}\right. \\
& \left.-2 \Psi_{y y} S_{x y}\right]+\beta\left\{\left[\frac{\partial^{2}}{\partial t^{2}}+\frac{\partial}{\partial t}\left(\Psi_{y} \frac{\partial}{\partial x}-\Psi_{x} \frac{\partial}{\partial y}\right)-2 \Psi_{x y t}\right.\right. \\
& -4 \Psi_{x y} \frac{\partial}{\partial t}+\left(\Psi_{y} \frac{\partial}{\partial x}-\Psi_{x} \frac{\partial}{\partial y}\right) \frac{\partial}{\partial t}+\left(\Psi_{y} \frac{\partial}{\partial x}-\Psi_{x} \frac{\partial}{\partial y}\right)
\end{aligned}
$$




$$
\begin{aligned}
& \left(\Psi_{y} \frac{\partial}{\partial x}-\Psi_{x} \frac{\partial}{\partial y}\right)-2\left(\Psi_{y} \Psi_{x x y}-\Psi_{x} \Psi_{x y y}\right)+4\left(\Psi_{x y}\right)^{2} \\
& \left.-2 \Psi_{y y} \Psi_{x x}-4 \Psi_{x y}\left(\Psi_{y} \frac{\partial}{\partial x}-\Psi_{x} \frac{\partial}{\partial y}\right)\right] S_{x x}+\left[-2 \Psi_{y y t}\right. \\
& -4 \Psi_{y y} \frac{\partial}{\partial t}+4 \Psi_{x y} \Psi_{y y}-2\left(\Psi_{y} \Psi_{x x y}-\Psi_{x} \Psi_{y y y}\right) \\
& \left.\left.-4 \Psi_{y y}\left(\Psi_{y} \frac{\partial}{\partial x}-\Psi_{x} \frac{\partial}{\partial y}\right)\right] S_{x y}-2 \Psi_{y y}^{2} S_{y y}\right\} \\
& =2 \Psi_{x y}+2 \lambda_{r}\left[\left(\frac{\partial}{\partial t}+\Psi_{y} \frac{\partial}{\partial x}-\Psi_{x} \frac{\partial}{\partial y}\right) \Psi_{x y}\right. \\
& \left.-2 \Psi_{x y}^{2}-\Psi_{y y}\left(\Psi_{y y}-\Psi_{x x}\right)\right], \\
& S_{x y}+\lambda\left[\left(\frac{\partial}{\partial t}+\Psi_{y} \frac{\partial}{\partial x}-\Psi_{x} \frac{\partial}{\partial y}\right) S_{x y}-\Psi_{x x} S_{x x}\right. \\
& \left.-\Psi_{y y} S_{y y}\right]+\beta\left\{\left[\frac{\partial^{2}}{\partial t^{2}}+\frac{\partial}{\partial t}\left(\Psi_{y} \frac{\partial}{\partial x}-\Psi_{x} \frac{\partial}{\partial y}\right)+\left(\Psi_{y} \frac{\partial}{\partial x}\right.\right.\right. \\
& \left.-\Psi_{x} \frac{\partial}{\partial y}\right) \frac{\partial}{\partial t}-4 \Psi_{y y} \Psi_{x x}+\left(\Psi_{y} \frac{\partial}{\partial x}-\Psi_{x} \frac{\partial}{\partial y}\right)\left(\Psi_{y} \frac{\partial}{\partial x}\right. \\
& \left.\left.-\Psi_{x} \frac{\partial}{\partial y}\right)\right] S_{x y}+\left[\Psi_{x x t}-\left(-\Psi_{y} \Psi_{x x x}+\Psi_{x} \Psi_{x x y}\right)\right. \\
& \left.+2 \Psi_{x x} \frac{\partial}{\partial t}+2 \Psi_{x x}\left(\Psi_{y} \frac{\partial}{\partial x}-\Psi_{x} \frac{\partial}{\partial y}\right)-2 \Psi_{x y} \Psi_{x x}\right] S_{x x} \\
& +\left[-\Psi_{y y t}-2 \Psi_{y y} \frac{\partial}{\partial t}-\left(\Psi_{y} \Psi_{x y y}-\Psi_{x} \Psi_{y y y}\right)-2 \Psi_{y y}\right. \\
& \left.\left.\left(\Psi_{y} \frac{\partial}{\partial x}-\Psi_{x} \frac{\partial}{\partial y}\right)-2 \Psi_{y y} \Psi_{x y}\right] S_{y y}\right\}=\left(\Psi_{y y}-\Psi_{x x}\right) \\
& +\lambda_{r}\left[\left(\frac{\partial}{\partial t}+\Psi_{y} \frac{\partial}{\partial x}-\Psi_{x} \frac{\partial}{\partial y}\right)\left(\Psi_{y y}-\Psi_{x x}\right)\right] \\
& +2 \lambda_{r} \Psi_{x y} \nabla^{2} \Psi, \\
& S_{y y}+\lambda\left[\left(\frac{\partial}{\partial t}+\Psi_{y} \frac{\partial}{\partial x}-\Psi_{x} \frac{\partial}{\partial y}\right) S_{y y}+2 \Psi_{x x} S_{x y}\right. \\
& \left.+2 \Psi_{x y} S_{y y}\right]+\beta\left\{\left[\frac{\partial^{2}}{\partial t^{2}}+\frac{\partial}{\partial t}\left(\Psi_{y} \frac{\partial}{\partial x}-\Psi_{x} \frac{\partial}{\partial y}\right)\right.\right. \\
& +2 \Psi_{x y t}+4 \Psi_{x y} \frac{\partial}{\partial t}+\left(\Psi_{y} \frac{\partial}{\partial x}-\Psi_{x} \frac{\partial}{\partial y}\right) \frac{\partial}{\partial t} \\
& +\left(\Psi_{y} \frac{\partial}{\partial x}-\Psi_{x} \frac{\partial}{\partial y}\right)\left(\Psi_{y} \frac{\partial}{\partial x}-\Psi_{x} \frac{\partial}{\partial y}\right)-2\left(-\Psi_{y} \Psi_{x x y}\right. \\
& \left.+\Psi_{x} \Psi_{x y y}\right)+4\left(\Psi_{x y}\right)^{2}-2 \Psi_{y y} \Psi_{x x}+4 \Psi_{x y}\left(\Psi_{y} \frac{\partial}{\partial x}\right.
\end{aligned}
$$

$$
\begin{aligned}
& \left.\left.-\Psi_{x} \frac{\partial}{\partial y}\right)\right] S_{y y}+\left[2 \Psi_{x x t}+4 \Psi_{x x} \frac{\partial}{\partial t}+4 \Psi_{x x} \Psi_{y y}\right. \\
& -2\left(-\Psi_{y} \Psi_{x x x}+\Psi_{x} \Psi_{x x y}\right)+4 \Psi_{x x}\left(\Psi_{y} \frac{\partial}{\partial x}\right. \\
& \left.\left.-\Psi_{x} \frac{\partial}{\partial y}\right)\right] S_{x y}+2 \Psi_{x x}^{2} S_{x x y} \\
& =-2 \Psi_{x y}+2 \lambda_{r}\left[-\left(\frac{\partial}{\partial t}+\Psi_{y} \frac{\partial}{\partial x}-\Psi_{x} \frac{\partial}{\partial y}\right) \Psi_{x y}\right. \\
& \left.-2 \Psi_{x y}^{2}+\Psi_{x x}\left(\Psi_{y y}-\Psi_{x x}\right)\right], \\
& \eta \quad \epsilon \cos \alpha(x-t), \\
& \Psi_{y}=0 \quad \text { and } \quad \Psi_{x}=\mp \alpha \epsilon \sin \alpha(x-t) \\
& \quad \text { at } y= \pm 1 \pm \eta, \\
& \frac{\partial}{\partial x}\left[m \frac{\partial^{2} \eta}{\partial t^{2}}+\frac{d}{R} \frac{\partial \eta}{\partial t}+\frac{B}{R^{2}} \frac{\partial^{4} \eta}{\partial x^{4}}-\frac{T}{R^{2}} \frac{\partial^{2} \eta}{\partial x^{2}}+\frac{K}{R^{2}} \eta\right] \\
& =\frac{1}{R}\left(S_{x x, x}+S_{x y, y}\right)-\left(\Psi_{y t}+\Psi_{y} \Psi_{y x}-\Psi_{x} \Psi_{y y}\right) \\
& \text { at } y= \pm 1 \pm \eta .
\end{aligned}
$$

In above equations $\epsilon(=a / h)$ is the amplitude ratio, $\alpha(=2 \pi h / \bar{\lambda})$ the wave number, $R(=c h / v)$ the Reynold number and $v(=\mu / \rho)$ the kinematic viscosity.

\section{Methodology of solution}

We expand the flow quantities in terms of $\epsilon$ as [22]

$$
\begin{aligned}
\Psi & =\Psi_{0}+\epsilon \Psi_{1}+\epsilon^{2} \Psi_{2}+\ldots \\
\frac{\partial p}{\partial x} & =\left(\frac{\partial p}{\partial x}\right)_{0}+\epsilon\left(\frac{\partial p}{\partial x}\right)_{1}+\epsilon^{2}\left(\frac{\partial p}{\partial x}\right)_{2}+\ldots, \\
S_{x x} & =S_{x x 0}+\epsilon S_{x x 1}+\epsilon^{2} S_{x x 2}+\ldots \\
S_{x y} & =S_{x y 0}+\epsilon S_{x y 1}+\epsilon^{2} S_{x y 2}+\ldots \\
S_{y y} & =S_{y y 0}+\epsilon S_{y y 1}+\epsilon^{2} S_{y y 2}+\ldots
\end{aligned}
$$

It is worth mentioning to point out that the first term on the right-hand side in Equation (23) corresponds to the imposed pressure gradient and the other terms correspond to the peristaltic motion. Using those equations 
into Equations (15)-(21) and then collecting terms of like powers of $\epsilon$, we obtain three sets of coupled differential equations with the corresponding boundary conditions in $\epsilon_{0}, \epsilon_{1}$ and $\epsilon_{2}$.

In actual practice $\epsilon_{0}$ set of differential equations with steady parallel flow and transverse symmetry assumption when pressure gradient is constant in the $x$-direction corresponds to the classical Poiseuille flow i.e.

$$
\begin{aligned}
\Psi_{0}(y) & =K_{0}\left[y-\frac{y^{3}}{3}\right]+c_{1}, \\
K_{0} & =-\frac{R}{2}\left(\frac{d p}{d x}\right)_{0},
\end{aligned}
$$

where $c_{1}$ is an arbitrary constant.

The second and third sets of differential equations in $\Psi_{1}$ and $\Psi_{2}$ can be expressed as follows:

$$
\begin{aligned}
& \Psi_{1}(x, y, t)=\frac{1}{2}\left[\Phi_{1}(y) e^{i \alpha(x-t)}+\Phi_{1}^{*}(y) e^{-i \alpha(x-t)}\right] \\
& S_{x x 1}=\frac{1}{2}\left[\Phi_{2}(y) e^{i \alpha(x-t)}+\Phi_{2}^{*}(y) e^{-i \alpha(x-t)}\right], \\
& S_{x y 1}=\frac{1}{2}\left[\Phi_{3}(y) e^{i \alpha(x-t)}+\Phi_{3}^{*}(y) e^{-i \alpha(x-t)}\right] \\
& S_{y y 1}=\frac{1}{2}\left[\Phi_{4}(y) e^{i \alpha(x-t)}+\Phi_{4}^{*}(y) e^{-i \alpha(x-t)}\right] \\
& \Psi_{2}(x, y, t)=\frac{1}{2}\left[\Phi_{20}(y)+\Phi_{22}(y) e^{2 i \alpha(x-t)}\right. \\
& \left.+\Phi_{22}^{*}(y) e^{-2 i \alpha(x-t)}\right]
\end{aligned}
$$$$
S_{x x 2}=\frac{1}{2}\left[\Phi_{30}(y)+\Phi_{33}(y) e^{2 i \alpha(x-t)}\right.
$$$$
\left.+\Phi_{33}^{*}(y) e^{-2 i \alpha(x-t)}\right]
$$$$
S_{x y 2}=\frac{1}{2}\left[\Phi_{40}(y)+\Phi_{44}(y) e^{2 i \alpha(x-t)}\right.
$$$$
\left.+\Phi_{44}^{*}(y) e^{-2 i \alpha(x-t)}\right],
$$$$
S_{y y 2}=\frac{1}{2}\left[\Phi_{50}(y)+\Phi_{55}(y) e^{2 i \alpha(x-t)}\right.
$$$$
\left.+\Phi_{55}^{*}(y) e^{-2 i \alpha(x-t)}\right] .
$$

Here asterisk indicates the complex conjugate. Invoking above equations into the differential equations and their corresponding boundary conditions in $\Psi_{1}$ and $\Psi_{2}$, we have three sets of coupled linear differential equations with their corresponding boundary conditions which are fourth-order ordinary differential equations with variable coefficients and the boundary conditions are not all homogeneous and the problem is not an eigenvalue problem. For free pumping case $(\partial p / \partial x)_{0}=0$ which means $K_{0}=0$. In this case we get

$$
\begin{aligned}
& i \alpha R\left[\frac{d^{2}}{d y^{2}}-\alpha^{2}\right] \Phi_{1}(y)+i \alpha \Phi_{2}^{\prime}(y)+\Phi_{3}^{\prime \prime}(y) \\
& +\alpha^{2} \Phi_{3}(y)-i \alpha \Phi_{4}^{\prime}(y)=0, \\
& \left(1-i \alpha \lambda-\alpha^{2} \beta\right) \Phi_{2}(y) \\
& =2 i \alpha\left(1-i \alpha \lambda_{r}\right) \Phi_{1}^{\prime}(y), \\
& \mu\left(1-i \alpha \lambda-\alpha^{2} \beta\right) \Phi_{3}(y) \\
& =\left(1-i \alpha \lambda_{r}\right)\left(\Phi_{1}^{\prime \prime}(y)+\alpha^{2} \Phi_{1}(y)\right), \\
& \mu\left(1-i \alpha \lambda-\alpha^{2} \beta\right) \Phi_{4}(y) \\
& =-2 i \alpha\left(1-i \alpha \lambda_{r}\right) \Phi_{1}^{\prime}(y), \\
& \Phi_{1}^{\prime}( \pm 1)=0, \\
& i \alpha R \Phi_{1}^{\prime}( \pm 1)+i \alpha \Phi_{2}( \pm 1)+\Phi_{3}^{\prime}( \pm 1)=R \delta, \\
& \delta=-\frac{i \alpha}{R^{2}}\left(\alpha^{2} R^{2} m+i \alpha R d-\alpha^{4} B-\alpha^{2} T-K\right), \\
& \Phi_{40}^{\prime \prime}(y) \\
& =\frac{i \alpha R}{2}\left[\Phi_{1}^{*}(y) \Phi_{1}^{\prime \prime}(y)-\Phi_{1}(y) \Phi_{1}^{* \prime \prime}(y)\right]^{\prime}, \\
& \Phi_{30}(y)=-\frac{i \alpha \lambda}{2}\left[\Phi_{1}^{*}(y) \Phi_{2}(y)-\Phi_{1}(y) \Phi_{2}^{*}(y)\right]^{\prime} \\
& +i \alpha \lambda\left[\Phi_{1}^{\prime}(y) \Phi_{2}^{*}(y)-\Phi_{2}(y) \Phi_{1}^{* \prime}(y)\right] \\
& +\lambda\left[\Phi_{1}^{\prime \prime}(y) \Phi_{3}^{*}(y)+\Phi_{1}^{* \prime \prime}(y) \Phi_{3}(y)\right] \\
& -\beta\left[\frac{3 \alpha^{2}}{2}\left(\Phi_{1}^{\prime}(y) \Phi_{2}^{*}(y)+\Phi_{2}(y) \Phi_{1}^{* \prime}(y)\right)\right. \\
& -\frac{\alpha^{2}}{2}\left(\Phi_{1}(y) \Phi_{2}^{\prime *}(y)+\Phi_{1}^{*}(y) \Phi_{2}^{\prime}(y)\right) \\
& \left.+2 i \alpha\left(\Phi_{1}^{\prime \prime}(y) \Phi_{3}^{*}(y)-\Phi_{1}^{* \prime \prime}(y) \Phi_{3}(y)\right)\right] \\
& -\alpha^{2} \lambda_{r}\left[\Phi_{1}(y) \Phi_{1}^{* \prime \prime}(y)+\Phi_{1}^{*}(y) \Phi_{1}^{\prime \prime}(y)\right.
\end{aligned}
$$




$$
\begin{aligned}
& \left.+6 \Phi_{1}^{\prime}(y) \Phi_{1}^{* \prime}(y)\right]-\lambda_{r}\left[\Phi _ { 1 } ^ { \prime \prime } ( y ) \left(\Phi_{1}^{* \prime \prime}(y)\right.\right. \\
& \left.+\alpha^{2} \Phi_{1}^{*}(y)\right)+\Phi_{1}^{* \prime \prime}(y)\left(\Phi_{1}^{\prime \prime}\right. \\
& \left.\left.(y)+\alpha^{2} \Phi_{1}(y)\right)\right] \text {, } \\
& \mp \frac{1}{2}\left[\Phi_{3}^{\prime \prime}( \pm 1)+\Phi_{3}^{* \prime \prime}( \pm 1)\right] \\
& -\frac{i \alpha R}{2}\left[\Phi_{1}( \pm 1) \Phi_{1}^{* \prime \prime}( \pm 1)\right. \\
& \left.-\Phi_{1}^{*}( \pm 1) \Phi_{1}^{\prime \prime}( \pm 1)\right] \\
& \mp \frac{i \alpha R}{2}\left[\Phi_{1}^{\prime \prime}( \pm 1)-\Phi_{1}^{* \prime \prime}( \pm 1)\right] \\
& \Phi_{40}(y)=\frac{i \alpha \lambda}{2}\left[\Phi_{1}(y) \Phi_{3}^{*}(y)-\Phi_{1}^{*}(y) \Phi_{3}(y)\right]^{\prime} \\
& +\frac{\alpha^{2} \lambda}{2}\left[\left(\Phi_{1}(y) \Phi_{2}^{*}(y)+\Phi_{1}^{*}(y) \Phi_{2}(y)\right)\right] \\
& +\frac{\lambda}{2}\left[\Phi_{1}^{\prime \prime}(y) \Phi_{4}^{*}(y)+\Phi_{1}^{* \prime \prime}(y) \Phi_{4}(y)\right] \\
& -\frac{i \alpha \lambda_{r}}{2}\left[\Phi_{1}(y) \Phi_{1}^{* \prime \prime}(y)-\Phi_{1}^{*}(y) \Phi_{1}^{\prime \prime}(y)\right]^{\prime} \\
& +i \alpha \lambda_{r}\left[\Phi_{1}^{\prime \prime}(y)\left(\Phi_{1}^{* \prime \prime}(y)-\alpha^{2} \Phi_{1}^{*}(y)\right)\right. \\
& \left.-\Phi_{1}^{* \prime \prime}(y)\left(\Phi_{1}^{\prime \prime}(y)-\alpha^{2} \Phi_{1}(y)\right)\right]+\Phi_{20}^{\prime \prime}(y) \\
& -\frac{\beta}{2}\left[\alpha^{2}\left(\Phi_{1}(y) \Phi_{3}^{*}(y)+\Phi_{1}^{*}(y) \Phi_{3}(y)\right)^{\prime}\right. \\
& -i \alpha^{3}\left(\Phi_{1}(y) \Phi_{2}^{*}(y)-\Phi_{1}^{*}(y) \Phi_{2}(y)\right) \\
& \left.-i \alpha\left(\Phi_{1}^{\prime \prime}(y) \Phi_{4}^{*}(y)-\Phi_{1}^{* \prime \prime}(y) \Phi_{4}(y)\right)\right], \\
& \Phi_{50}(y)=\frac{i \alpha \lambda}{2}\left[\Phi_{1}(y) \Phi_{4}^{*}(y)-\Phi_{1}^{*}(y) \Phi_{4}(y)\right]^{\prime} \\
& -i \alpha \lambda\left[\Phi_{1}^{\prime}(y) \Phi_{4}^{*}(y)-\Phi_{1}^{\prime *}(y) \Phi_{4}(y)\right] \\
& -\frac{\beta}{2}\left[\alpha^{2}\left(\Phi_{1}(y) \Phi_{4}^{*}(y)+\Phi_{1}^{*}(y) \Phi_{4}(y)\right)^{\prime}\right. \\
& -2 \alpha^{2}\left(\Phi_{1}^{\prime}(y) \Phi_{4}^{*}(y)+\Phi_{1}^{\prime *}(y) \Phi_{4}(y)\right) \\
& \left.-2 i \alpha^{3}\left(\Phi_{1}(y) \Phi_{3}^{*}(y)-\Phi_{1}^{*}(y) \Phi_{3}(y)\right)\right] \\
& +\alpha^{2} \lambda\left[\left(\Phi_{1}(y) \Phi_{3}^{*}(y)+\Phi_{1}^{*}(y) \Phi_{3}(y)\right)\right] \\
& -2 \alpha^{2} \lambda_{r}\left[\Phi_{1}^{\prime}(y) \Phi_{1}^{\prime *}(y)+\alpha^{2} \Phi_{1}(y) \Phi_{1}^{*}(y)\right], \\
& 2 i \alpha R\left[\frac{d^{2}}{d y^{2}}-4 \alpha^{2}\right] \Phi_{22}(y) \\
& =\frac{i \alpha R}{2}\left[\Phi_{1}^{\prime}(y) \Phi_{1}^{\prime \prime}(y)-\Phi_{1}(y) \Phi_{1}^{\prime \prime \prime}(y)\right] \\
& -\Phi_{44}^{\prime \prime}(y)-4 \alpha^{2} \Phi_{44}(y) \\
& -2 i \alpha \Phi_{33}^{\prime}(y)+2 i \alpha \Phi_{55}^{\prime}(y), \\
& (1-2 i \alpha \lambda) \Phi_{33}(y) \\
& =\frac{i \alpha \lambda}{2}\left(\Phi_{1}(y) \Phi_{2}(y)\right)^{\prime}+\lambda \Phi_{1}^{\prime \prime}(y) \Phi_{3}(y) \\
& -\beta\left[-4 \alpha^{2} \Phi_{33}(y)-\frac{3 \alpha^{2}}{2}\left(\Phi_{1}(y) \Phi_{2}(y)\right)^{\prime}\right. \\
& \left.+3 i \alpha \Phi_{1}^{\prime \prime}(y) \Phi_{3}(y)\right] \\
& -\lambda_{r} \Phi_{1}^{\prime \prime}(y)\left(\Phi_{1}^{\prime \prime}(y)+\alpha^{2} \Phi_{1}(y)\right) \\
& +4 i \alpha\left(1-2 i \alpha \lambda_{r}\right) \Phi_{22}^{\prime}(y) \\
& +\alpha^{2} \lambda_{r}\left(\Phi_{1}(y) \Phi_{1}^{\prime}(y)\right)^{\prime}, \\
& (1-2 i \alpha \lambda) \Phi_{44}(y) \\
& =\frac{i \alpha \lambda}{2}\left[\Phi_{1}^{\prime}(y) \Phi_{3}(y)-\Phi_{1}(y) \Phi_{3}^{\prime}(y)\right] \\
& +\frac{\alpha^{2} \lambda}{2}\left(\Phi_{1}(y) \Phi_{2}(y)\right)+\frac{\lambda}{2} \Phi_{1}^{\prime \prime}(y) \Phi_{4}(y) \\
& -\beta\left[-4 \alpha^{2} \Phi_{44}(y)+\frac{3 \alpha^{2}}{2}\left(\Phi_{1}^{\prime}(y) \Phi_{3}(y)\right.\right. \\
& \left.-\Phi_{1}(y) \Phi_{3}^{\prime}(y)\right)+\frac{3 i \alpha^{3}}{2} \Phi_{1}(y) \Phi_{2}(y) \\
& \left.+\frac{3 i \alpha}{2} \Phi_{1}^{\prime \prime}(y) \Phi_{4}(y)\right]+\left(1-2 i \alpha \lambda_{r}\right) \\
& \left(\Phi_{22}^{\prime \prime}(y)+4 \alpha^{2} \Phi_{22}(y)\right) \\
& +\frac{i \alpha \lambda_{r}}{2}\left(3 \Phi_{1}^{\prime}(y) \Phi_{1}^{\prime \prime}(y)\right.
\end{aligned}
$$




$$
\begin{aligned}
&\left.-\Phi_{1}(y) \Phi_{1}^{\prime \prime \prime}(y)\right) \\
&-i \alpha^{3} \lambda_{r}\left(\Phi_{1}(y) \Phi_{1}^{\prime}(y)\right) \\
&(1-2 i \alpha \lambda) \Phi_{55}(y) \\
&= \frac{i \alpha \lambda}{2}\left[\Phi_{1}(y) \Phi_{4}^{\prime}(y)-3 \Phi_{1}^{\prime}(y) \Phi_{4}(y)\right] \\
&+\alpha^{2} \lambda \Phi_{1}(y) \Phi_{3}(y)-\beta\left[-4 \alpha^{2} \Phi_{55}(y)\right. \\
&+\frac{3 \alpha^{2}}{2}\left(\Phi_{1}^{\prime}(y) \Phi_{4}(y)-\Phi_{1}(y) \Phi_{4}^{\prime}(y)\right)+3 \alpha^{2} \\
&\left.\Phi_{1}^{\prime}(y) \Phi_{4}(y)+3 i \alpha^{3} \Phi_{1}(y) \Phi_{3}(y)\right] \\
&+\alpha^{2} \lambda_{r}\left[3 \Phi_{1}^{\prime}(y) \Phi_{1}^{\prime}(y)-2 \Phi_{1}(y) \Phi_{1}^{\prime \prime}(y)\right. \\
&\left.-\alpha^{2} \Phi_{1}^{2}(y)\right]-4 i \alpha\left(1-2 i \alpha \lambda_{r}\right) \Phi_{22}^{\prime}(y), \\
& \Phi_{22}^{\prime}( \pm 1)=\mp \frac{1}{2} \Phi_{1}^{\prime \prime}( \pm 1),
\end{aligned}
$$$$
2 \Phi_{44}^{\prime}( \pm 1)
$$$$
=+i \alpha R \Phi_{1}^{\prime}( \pm 1) \Phi_{1}^{\prime}( \pm 1)-4 i \alpha \Phi_{33}( \pm 1)
$$$$
\mp \Phi_{3}^{\prime \prime}( \pm 1) \mp i \alpha \Phi_{2}^{\prime}( \pm 1)-4 i \alpha R \Phi_{22}^{\prime}( \pm 1)
$$$$
\mp i \alpha R \Phi_{1}^{\prime \prime}( \pm 1)-i \alpha R \Phi_{1}( \pm 1) \Phi_{1}^{\prime \prime}( \pm 1) \text {, }
$$

in which primes denote differentiation with respect to $y$.

Equations (36)-(41) have the solutions in the following forms

$$
\begin{aligned}
& \Phi_{1}(y)=A_{1} \sinh \alpha y+B_{1} \sinh \tau y, \\
& \Phi_{2}(y)=A_{2} \cosh \alpha y+B_{2} \cosh \tau y, \\
& \Phi_{3}(y)=A_{3} \sinh \alpha y+B_{3} \sinh \tau y, \\
& \Phi_{4}(y)=-A_{2} \cosh \alpha y-B_{2} \cosh \tau y,
\end{aligned}
$$

where

$$
\begin{aligned}
& A_{1}=-\frac{i \delta}{\alpha^{2} \cosh \alpha}, \quad B_{1}=\frac{i \delta}{\alpha \tau \cosh \tau}, \\
& A_{2}=2 i \alpha^{2} \Gamma A_{1}, \quad B_{2}=2 i \alpha \tau \Gamma B_{1}, \\
& A_{3}=2 \alpha^{2} \Gamma A_{1}, \quad B_{3}=\left(\alpha^{2}+\tau^{2}\right) \Gamma B_{1}, \\
& \tau^{2}=\alpha^{2}-\frac{i \alpha R}{\Gamma}, \quad \Gamma=\frac{\left(1-i \alpha \lambda_{r}\right)}{\left(1-i \alpha \lambda-\alpha^{2} \beta\right)}
\end{aligned}
$$

and solution of Equations (42)-(47) satisfies the following expression

$$
\Phi_{20}^{\prime}(y)=F(y)-F(1)+D-C_{1}\left(1-y^{2}\right) .
$$

The peristaltic mean flow is

$$
\begin{aligned}
\bar{u}(y) & =\frac{\epsilon^{2}}{2} \Phi_{20}^{\prime}(y) \\
& =\frac{\epsilon^{2}}{2}\left[F(y)-F(1)+D-C_{1}\left(1-y^{2}\right)\right],
\end{aligned}
$$

whence

$$
\begin{aligned}
& D=-\frac{1}{2}\left[\left(A_{1}+A_{1}^{*}\right) \alpha^{2} \sinh \alpha\right. \\
& \left.+B_{1} \tau^{2} \sinh \tau+B_{1}^{*} \tau^{* 2} \sinh \tau^{*}\right], \\
& C_{1}=\frac{\left(A_{11}+A_{11}^{*}\right)}{2} \sinh \alpha \\
& +\frac{B_{11}}{2} \sinh \tau+\frac{B_{11}^{*}}{2} \sinh \tau^{*}, \\
& A_{11}=\frac{\alpha^{2}}{2}\left[-i \alpha R A_{1}-i A_{2}-\alpha A_{3}\right] \text {, } \\
& B_{11}=-\frac{\tau}{2}\left[i \alpha R \tau B_{1}+i \alpha B_{2}+\tau B_{3}\right], \\
& F(y)=s_{1} \cosh 2 \alpha y+s_{2} \cosh (\alpha+\tau) y \\
& +s_{3} \cosh (\alpha-\tau) y+s_{4} \cosh \left(\alpha+\tau^{*}\right) y \\
& +s_{5} \cosh \left(\alpha-\tau^{*}\right) y \\
& +s_{6} \cosh \left(\tau+\tau^{*}\right) y \\
& +s_{7} \cosh \left(\tau-\tau^{*}\right) y, \\
& s_{1}=\frac{i \alpha(\lambda+i \alpha \beta)}{4}\left(A_{1}^{*} A_{3}-A_{1} A_{3}^{*}\right), \\
& s_{2}=\frac{i \alpha R(\tau-\alpha) A_{1}^{*} B_{1}}{4(\tau+\alpha)}-\frac{i \alpha(\lambda+i \alpha \beta)}{4} \\
& \left(A_{3}^{*} B_{1}-A_{1}^{*} B_{3}\right)-\frac{(\lambda+i \alpha \beta)}{4}(\alpha-\tau) B_{1} A_{2}^{*} \\
& -\frac{i \alpha \lambda_{r}}{4}(\tau-\alpha)^{2} A_{1}^{*} B_{1}, \\
& s_{3}=\frac{i \alpha R(\alpha+\tau) A_{1}^{*} B_{1}}{4(\alpha-\tau)}+\frac{i \alpha(\lambda+i \alpha \beta)}{4} \\
& \left(A_{3}^{*} B_{1}-A_{1}^{*} B_{3}\right)+\frac{(\lambda+i \alpha \beta)}{4}(\alpha+\tau) B_{1} A_{2}^{*} \\
& +\frac{i \alpha \lambda_{r}}{4}(\alpha+\tau)^{2} A_{1}^{*} B_{1} \\
& s_{4}=\frac{i \alpha R\left(\alpha-\tau^{*}\right) A_{1} B_{1}^{*}}{4\left(\alpha+\tau^{*}\right)}-\frac{i \alpha(\lambda+i \alpha \beta)}{4}
\end{aligned}
$$




$$
\begin{aligned}
& \left(A_{1} B_{3}^{*}-B_{1}^{*} A_{3}\right)-\frac{(\lambda-i \alpha \beta)}{4}\left(\alpha-\tau^{*}\right) B_{1}^{*} A_{2} \\
& +\frac{i \alpha \lambda_{r}}{4}\left(\alpha-\tau^{*}\right)^{2} A_{1} B_{1}^{*}, \\
& s_{5}=-\frac{i \alpha R\left(\alpha+\tau^{*}\right) A_{1} B_{1}^{*}}{4\left(\alpha-\tau^{*}\right)}+\frac{i \alpha(\lambda+i \alpha \beta)}{4} \\
& \left(A_{1} B_{3}^{*}-B_{1}^{*} A_{3}\right)+\frac{(\lambda-i \alpha \beta)}{4}\left(\alpha+\tau^{*}\right) B_{1}^{*} A_{2} \\
& -\frac{i \alpha \lambda_{r}}{4}\left(\alpha+\tau^{*}\right)^{2} A_{1} B_{1}^{*}, \\
& s_{6}=\frac{i \alpha R\left(\tau-\tau^{*}\right) B_{1} B_{1}^{*}}{4\left(\tau+\tau^{*}\right)}-\frac{i \alpha(\lambda+i \alpha \beta)}{4} \\
& \left(B_{1} B_{3}^{*}-B_{1}^{*} B_{3}\right)-\frac{(\lambda+i \alpha \beta)\left(\alpha^{2}-\tau^{2}\right)}{4\left(\tau+\tau^{*}\right)} B_{1} B_{2}^{*} \\
& -\frac{(\lambda-i \alpha \beta)\left(\alpha^{2}-\tau^{* 2}\right)}{4\left(\tau+\tau^{*}\right)} B_{1}^{*} B_{2} \\
& +\frac{i \alpha \lambda_{r}\left(\tau-\tau^{*}\right) B_{1} B_{1}^{*}}{4\left(\tau+\tau^{*}\right)}\left(2 \alpha^{2}-\tau^{2}-\tau^{* 2}\right), \\
& s_{7}=-\frac{i \alpha R\left(\tau+\tau^{*}\right) B_{1} B_{1}^{*}}{4\left(\tau-\tau^{*}\right)}+\frac{i \alpha(\lambda+i \alpha \beta)}{4} \\
& \left(B_{1} B_{3}^{*}-B_{1}^{*} B_{3}\right)-\frac{(\lambda+i \alpha \beta)\left(\alpha^{2}-\tau^{2}\right)}{4\left(\tau-\tau^{*}\right)} B_{1} B_{2}^{*} \\
& +\frac{(\lambda-i \alpha \beta)\left(\alpha^{2}-\tau^{* 2}\right)}{4\left(\tau-\tau^{*}\right)} B_{1}^{*} B_{2} \\
& +\frac{i \alpha \lambda_{r}\left(\tau+\tau^{*}\right) B_{1} B_{1}^{*}}{4\left(\tau^{*}-\tau\right)}\left(2 \alpha^{2}-\tau^{2}-\tau^{* 2}\right),
\end{aligned}
$$

The critical reflux condition $\bar{u}$ is zero at $y=0$ [17] and so substituting Equation (59) we arrive at

$$
\begin{gathered}
T_{\text {critical reflux }}=\frac{H_{5}+\sqrt{H_{5}^{2}-4 H_{6}}}{2}, \\
H=\left[\frac{h_{1}}{2 \alpha^{4} \cosh ^{2} \alpha}+\frac{\left(h_{2}+h_{3}\right)}{4 \alpha^{3} \tau \cosh \alpha \cosh \tau}\right. \\
\left.+\frac{\left(h_{4}+h_{5}\right)}{4 \alpha^{3} \tau^{*} \cosh \alpha \cosh \tau^{*}}+\frac{\left(h_{6}+h_{7}\right)}{4 \alpha^{2} \tau \tau^{*} \cosh \tau \cosh \tau^{*}}\right], \\
H_{1}=\frac{\alpha^{2}}{2}\left[2 \alpha \tanh \alpha-\tau \tanh \tau-\tau^{*} \tanh \tau^{*}\right. \\
\left.+\frac{i \alpha}{2}\left(L_{5} \tau \tanh \tau-L_{5}^{*} \tau^{*} \tanh \tau^{*}\right)\right],
\end{gathered}
$$

$$
\begin{aligned}
H_{2}= & \frac{1}{2}\left[\left(L_{4}+L_{4}^{*}\right) \alpha \tanh \alpha\right. \\
& \left.-L_{4} \tau \tanh \tau-L_{4}^{*} \tau^{*} \tanh \tau^{*}\right], \\
H_{3}= & -\frac{i \alpha}{4}\left[\alpha R\left(L_{4}-L_{4}^{*}\right) \tanh \alpha-L_{4} L_{5} \tau \tanh \tau\right. \\
& \left.-L_{4}^{*} L_{5}^{*} \tau^{*} \tanh \tau^{*}\right], H_{4}=H_{2}+H_{3}, \\
H_{5}= & \frac{R^{2}}{\alpha^{6} H}\left[\frac{2 \alpha^{4} H L_{3}}{R^{2}}+H_{1}\right], \\
H_{6}= & \frac{R^{2}}{\alpha^{6} H}\left[\frac{\alpha^{2} H L_{4} L_{4}^{*}}{R^{2}}+H_{4}\right], \\
L_{3}= & \alpha^{2} R^{2} m-\alpha^{4} B-K, \\
L_{4}= & i \alpha R d+L_{3}, \\
L_{5}= & {\left[R+2 i \alpha \Gamma-\frac{i \Gamma}{\alpha}\left(\alpha^{2}+\tau^{2}\right)\right], } \\
h_{1}= & i \alpha^{3}(\lambda+i \alpha \beta)(1-\cosh 2 \alpha)\left(\Gamma-\Gamma^{*}\right), \\
h_{2}= & {[1-\cosh (\alpha+\tau)] i \alpha\left\{\frac{R(\alpha-\tau)}{(\alpha+\tau)}\right.} \\
& +(\lambda+i \alpha \beta)\left[-\Gamma\left(\alpha^{2}+\tau^{2}\right)+2 \alpha^{2} \Gamma^{*}\right] \\
h_{3}= & {[1-\cosh (\alpha-\tau)] i \alpha\left\{-\frac{R(\alpha+\tau)}{(\alpha-\tau)}\right.} \\
& -(\lambda+i \alpha \beta)\left[-\Gamma\left(\alpha^{2}+\tau^{2}\right)+2 \alpha^{2} \Gamma^{*}\right] \\
& \\
& \left.\left.-2 \alpha \beta \Gamma^{*}(\alpha-\tau)+\lambda\right)-\lambda r(\alpha+\tau)^{2}\right\},
\end{aligned}
$$$$
\begin{aligned}
h_{4}=[1 & \left.-\cosh \left(\alpha+\tau^{*}\right)\right] i \alpha\left\{\frac{-R\left(\alpha-\tau^{*}\right)}{\left(\alpha+\tau^{*}\right)}\right. \\
& +(\lambda+i \alpha \beta)\left[\Gamma^{*}\left(\alpha^{2}+\tau^{* 2}\right)-2 \alpha^{2} \Gamma\right] \\
& \left.+2 \alpha(\lambda+i \alpha \beta) \Gamma\left(\alpha-\tau^{*}\right)-\lambda_{r}\left(\alpha-\tau^{*}\right)^{2}\right\},
\end{aligned}
$$$$
h_{5}=\left[1-\cosh \left(\alpha-\tau^{*}\right)\right] i \alpha\left\{\frac{R\left(\alpha+\tau^{*}\right)}{\left(\alpha-\tau^{*}\right)}\right.
$$$$
+(\lambda+i \alpha \beta)\left[-\Gamma^{*}\left(\alpha^{2}+\tau^{* 2}\right)+2 \alpha^{2} \Gamma\right]
$$$$
\left.-2 \alpha(\lambda-i \alpha \beta) \Gamma\left(\alpha+\tau^{*}\right)+\lambda_{r}\left(\alpha+\tau^{*}\right)^{2}\right\} \text {, }
$$ 


$$
\begin{aligned}
h_{6}= & {\left[1-\cosh \left(\tau+\tau^{*}\right)\right] i \alpha\left\{\frac{R\left(\tau-\tau^{*}\right)}{\left(\tau+\tau^{*}\right)}\right.} \\
& -(\lambda+i \alpha \beta)\left[-\Gamma\left(\alpha^{2}+\tau^{2}\right)+\Gamma^{*}\left(\alpha^{2}+\tau^{* 2}\right)\right] \\
& +2(\lambda+i \alpha \beta) \tau^{*} \Gamma^{*} \frac{\left(\alpha^{2}-\tau^{2}\right)}{\left(\tau+\tau^{*}\right)} \\
& -2(\lambda-i \alpha \beta) \tau \Gamma \frac{\left(\alpha^{2}-\tau^{* 2}\right)}{\left(\tau+\tau^{*}\right)} \\
& \left.+\lambda_{r} \frac{\left(2 \alpha^{2}-\tau^{2}-\tau^{* 2}\right)\left(\tau-\tau^{*}\right)}{\left(\tau+\tau^{*}\right)}\right\},
\end{aligned}
$$

$$
\begin{aligned}
h_{7}= & {\left[1-\cosh \left(\tau-\tau^{*}\right)\right] i \alpha\left\{-\frac{R\left(\tau+\tau^{*}\right)}{\left(\tau-\tau^{*}\right)}\right.} \\
& +(\lambda+i \alpha \beta)\left[-\Gamma\left(\alpha^{2}+\tau^{2}\right)+\Gamma^{*}\left(\alpha^{2}+\tau^{* 2}\right)\right] \\
& +2(\lambda+i \alpha \beta) \tau^{*} \Gamma^{*} \frac{\left(\alpha^{2}-\tau^{2}\right)}{\left(\tau-\tau^{*}\right)} \\
& +2(\lambda-i \alpha \beta) \tau \Gamma \frac{\left(\alpha^{2}-\tau^{* 2}\right)}{\left(\tau-\tau^{*}\right)} \\
& \left.+\lambda_{r} \frac{\left(2 \alpha^{2}-\tau^{2}-\tau^{* 2}\right)\left(\tau+\tau^{*}\right)}{\left(\tau^{*}-\tau\right)}\right\} .
\end{aligned}
$$
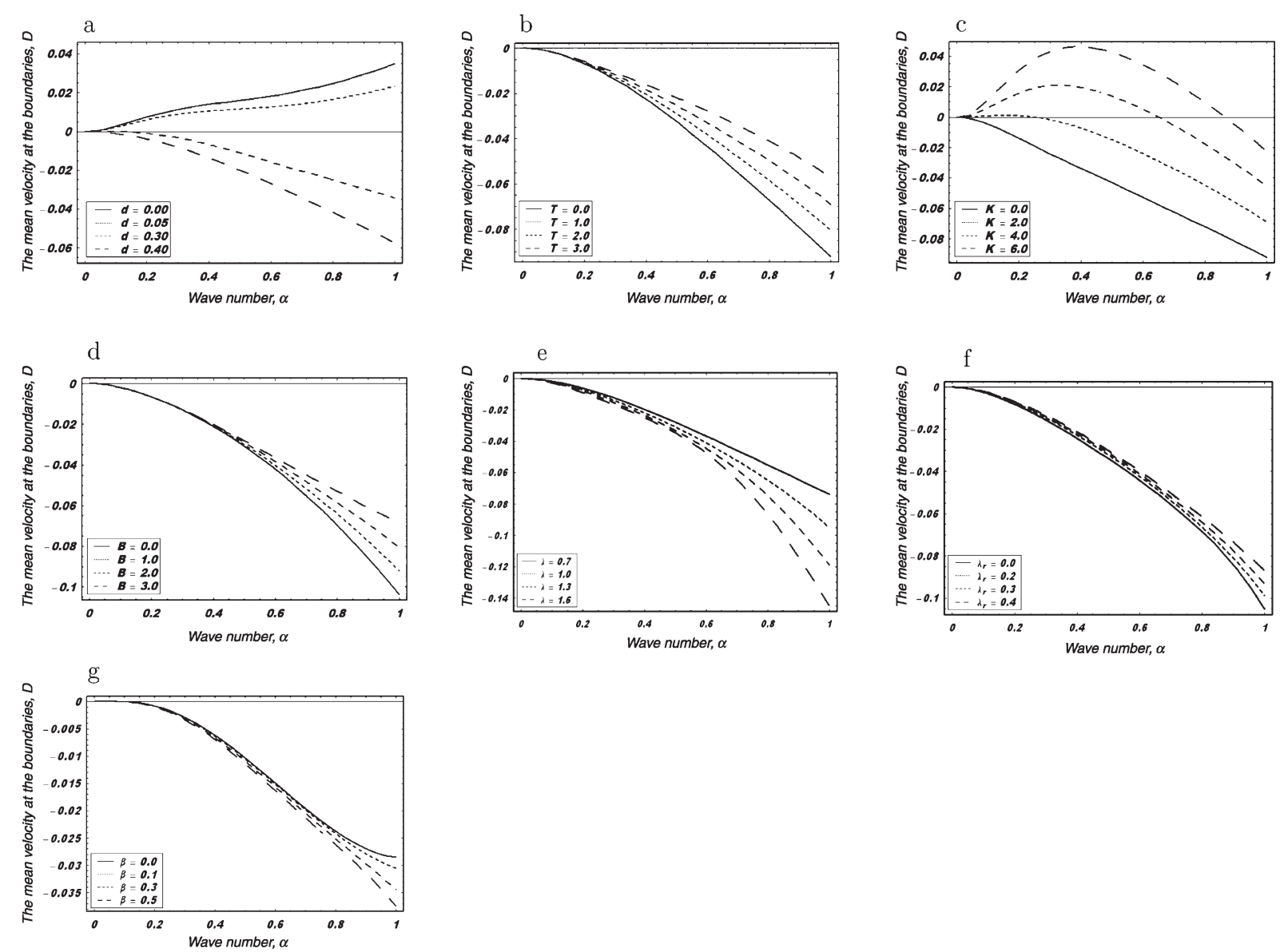

Fig. 1. The variation of $D$ with wave number $\alpha$ for four different values of wall damping $d$ (panel $(a)$ ), wall tension $T$ (panel $(b)$ ), spring stiffness $K(\operatorname{panel}(c))$, flexural rigidity $B(\operatorname{panel}(d))$, relaxation time $\lambda(\operatorname{panel}(e))$, retardation time $\lambda_{r}(\operatorname{panel}(f))$ and Burgers' fluid parameter $\beta$ (panel $(g)$ ). The other parameters chosen are $m=0.01, B=2, T=1, K=1, R=10, \lambda=0.8, \lambda_{r}=0.5$ and $\beta=0.3(\operatorname{panel}(a)) ; m=0.01$, $B=2, K=1, R=10, d=0.5, \lambda=0.8, \lambda_{r}=0.5$ and $\beta=0.3(\operatorname{panel}(b)) ; m=0.01, B=2, T=1, R=10, d=0.5, \lambda=0.8, \lambda_{r}=0.5$ and $\beta=0.3(\operatorname{panel}(c)) ; m=0.01, T=1, K=1, R=10, d=0.5, \lambda=0.8, \lambda_{r}=0.5$ and $\beta=0.3(\operatorname{panel}(d)) ; m=0.01, B=2, T=1, K=1$, $R=10, d=0.5, \lambda_{r}=0.5$ and $\beta=0.3(\operatorname{panel}(e)) ; m=0.01, B=2, T=1, K=1, R=10, d=0.5, \lambda=0.8$ and $\beta=0.3(\operatorname{panel}(f))$ and $m=0.01, d=0.3, B=2, T=1, K=1, R=10, \lambda=0.8$ and $\lambda_{r}=0.5(\operatorname{panel}(g))$. 


\section{Results and discussion}

This section deals with the variation of various important parameters by plotting graphs. Particularly the mean velocity at the channel boundaries $D$, and the time-averaged mean axial velocity distribution and reversal flow are computed when $K_{0}=0$. In fact the constant $D$ initially arises due to the no-slip condition of the axial-velocity on the wall because of the value of $\Phi_{20}^{\prime}(y)$ at the boundary. The mean-velocity at the boundaries of the channel is $\bar{u}( \pm 1)=\frac{\epsilon^{2}}{2} \Phi_{20}^{\prime}( \pm 1)=$ $\frac{\epsilon^{2}}{2} D$ [17]. The constant $\frac{\epsilon^{2}}{2} D$ depicts the mean velocity at the channel boundaries. The behavior of wall damping $d$ on the boundaries $D$ is sketched in Fig. 1a. It is found that $D$ decreases when $d$ is increased. The choice $d=0$ implies that the wall moves up and down with no damping force on it and therefore indicates the case of elastic walls. The variation of $T$ on $D$ with $\alpha$ is shown in Fig. 1b. Here $D$ is an increasing function of $T$. Fig. 1c and d elucidates the effect of spring stiffness $K$ and flexural rigidity of the plate $B$ respectively on $D$. It is observed that the mean velocity at the boundaries

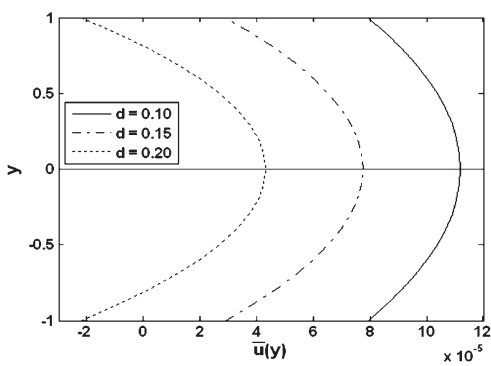

d

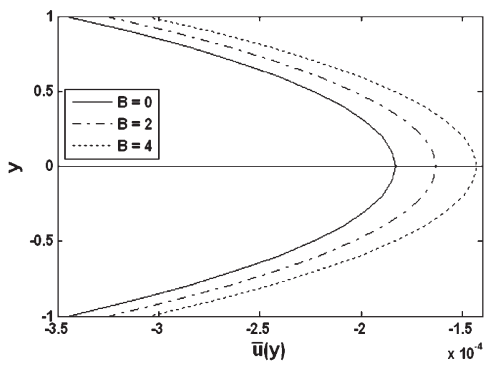

$\mathrm{g}$



$\mathrm{b}$
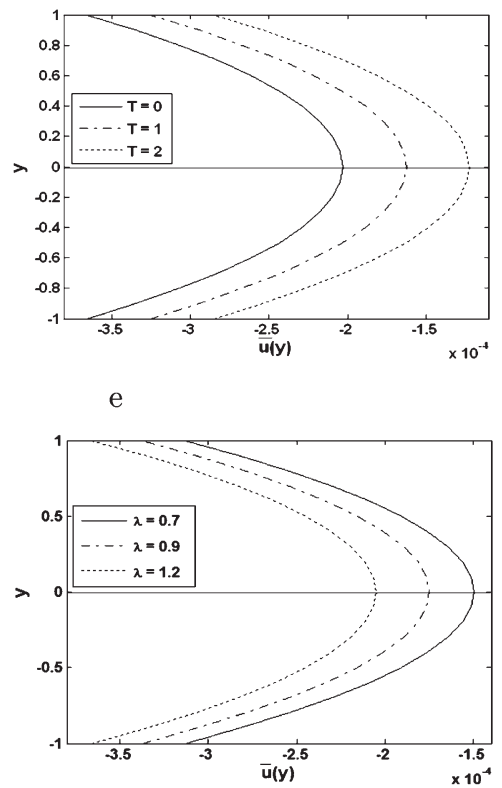

$\mathrm{h}$

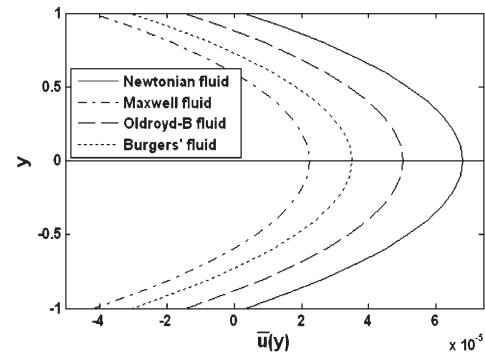

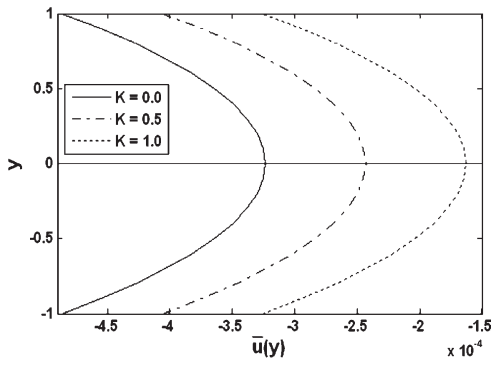

f

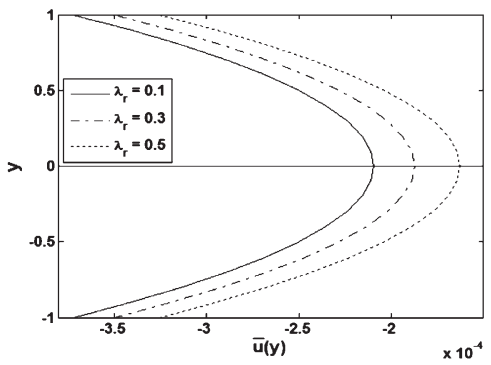

Fig. 2. The variation of mean-velocity distribution and reversal flow for three different values of wall damping $d$ (panel $(a)$ ), wall tension $T$ (panel $(b))$, spring stiffness $K(\operatorname{panel}(c))$, flexural rigidity $B(\operatorname{panel}(d))$, relaxation time $\lambda(\operatorname{panel}(e))$, retardation time $\lambda_{r}($ panel $(f))$ and Burgers' fluid parameter $\beta$ (panel $(g))$. The other parameters chosen are $m=0.01, B=2, T=1, K=1, R=10, \alpha=0.5, \epsilon=0.15, \lambda=0.8, \lambda r=0.5$ and $\beta=0.3(\operatorname{panel}(a)) ; m=0.01, B=2, K=1, R=10, d=0.5, \alpha=0.5, \epsilon=0.15, \lambda=0.8, \lambda=0.5$ and $\beta=0.3(\operatorname{panel}(b)) ; m=0.01$, $B=2, T=1, d=0.5, R=10, \alpha=0.5, \epsilon=0.15, \lambda=0.8, \lambda_{r}=0.5$ and $\beta=0.3(\operatorname{panel}(c)) ; m=0.01, d=0.5, T=1, K=1, R=10$, $\alpha=0.5, \epsilon=0.15, \lambda=0.8, \lambda_{r}=0.5$ and $\beta=0.3(\operatorname{panel}(d)) ; m=0.01, B=2, T=1, K=1, R=10, d=0.5, \alpha=0.5, \epsilon=0.15, \lambda=0.5$ and $\beta=0.3(\operatorname{panel}(e)) ; m=0.01, B=2, T=1, K=1, R=10, d=0.5, \alpha=0.5, \epsilon=0.15, \lambda=0.8$ and $\beta=0.3(\operatorname{panel}(f))$ and $m=0.01$, $B=2, T=1, K=1, R=10, d=0.2, \alpha=0.5, \epsilon=0.15, \lambda=0.8$ and $\lambda_{r}=0.5(\operatorname{panel}(g))$. 

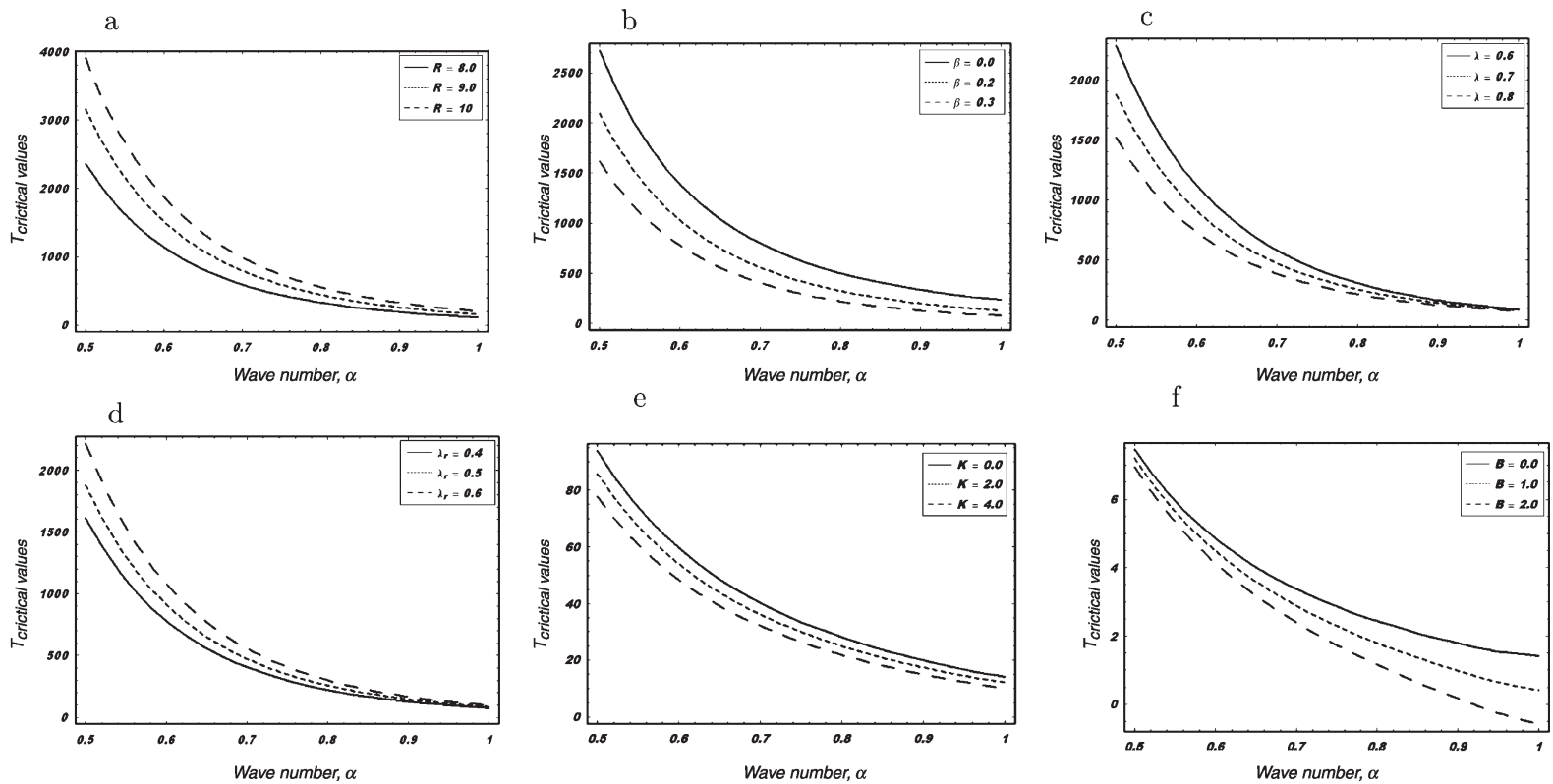

Fig. 3. The variation of critical values of the wall tension $T$ with wave number $\alpha$ for three different values of the Reynolds number $R$ (panel ( $a$ )), Burgers' fluid parameter $\beta$ (panel $(b))$, relaxation time $\lambda(\operatorname{panel}(c))$, retardation time $\lambda_{r}(\operatorname{panel}(d))$, spring stiffness $K(\operatorname{panel}(e))$ and flexural rigidity $B(\operatorname{panel}(f))$. The other parameters chosen are $m=0.01, B=2, K=1, d=0.5, \lambda=0.7, \lambda_{r}=0.4$ and $\beta=0.3(\operatorname{panel}(a)) ; m=0.01$, $R=7, B=2, K=1, d=0.5, \lambda=0.7$ and $\lambda_{r}=0.4(\operatorname{panel}(b)) ; m=0.01, B=2, K=1, d=0.5, R=7, \lambda_{r}=0.5$ and $\beta=0.3(\operatorname{panel}(c))$ $m=0.01, B=2, K=1, d=0.5, R=7, \lambda=0.7$ and $\beta=0.3(\operatorname{panel}(d)) ; m=0.01, R=5, B=2, d=0.5, \beta=0.3, \lambda=2$ and $\lambda=0.7$ $(\operatorname{panel}(e))$ and $m=0.01, R=3, K=1, d=0.5, \beta=0.3, \lambda=1.5$ and $\lambda_{r}=0.5(\operatorname{panel}(f))$.

increases in these figures. The effects of relaxation $(\lambda)$ and retardation $\left(\lambda_{r}\right)$ times on the mean velocity at the boundaries are shown in Fig. 1e and f respectively. It is revealed that the mean velocity decreases when $\lambda$ is increased. However opposite trend of mean velocity is observed when $\lambda_{r}$ is increased. Fig. $1 \mathrm{~g}$ shows the effect of the Burgers' fluid parameter $\beta$ on the mean velocity at the boundaries of the channel. This Figure depicts that the value of $D$ decreases by increasing $\beta$. After analyzing the effects of wall properties, which arise due to different considerations, separately, we intend to learn the effects on the velocity $\bar{u}(y)$. Expression (59) for mean axial velocity $(\bar{u}(y))$ shows that it is dominated by the constant $D$ and a parabolic distribution $-C_{1}\left(1-y^{2}\right)$. The effects of $d$ on the mean velocity distribution and reversal flow are presented in Fig. $2 \mathrm{a}$. It is shown that the velocity decreases by increasing $d$, while in case of $T$ the situation is quite opposite (Fig. 2b). Physically it means that dampness gives an adverse effect on the velocity while it is favored by wall tension. The effects of spring stiffness coefficient $(K)$ and flexural rigidity of the plate $(B)$ are presented in Fig. 2c and d respectively. It is depicted that the magnitude of velocity is an increasing function of $K$ and $B$. It means that wall elastance and rigidity gives favorable effects on the velocity. Fig. $2 \mathrm{e}$ and $\mathrm{f}$ show the opposite effects of relaxation time $\lambda$ and the retardation time $\lambda_{r}$ respectively, on the mean velocity distribution. These Figs. describe that the magnitude of the velocity $\bar{u}(y)$ increases upon increasing $\lambda$ while the reverse situation is noted in the case of $\lambda_{r}$. Fig. $2 \mathrm{~g}$ displays that $\bar{u}(y)$ is a decreasing function of $\beta$. Fig. $2 \mathrm{~h}$ represents the effect of different fluids on the time-averaged mean axial velocity distribution and reversal flow. This Fig. shows that velocity is maximum in case of viscous fluid when compared with the non-Newtonian fluids. This is due to the presence of the viscoelastic parameters that are involved in the non-Newtonian fluids. Also the numerical values of $\bar{u}(y)$ for different values of the parameters are presented in Table 1. This table shows that the viscous fluid possesses maximum velocity and the Maxwell fluid has the minimum velocity. Moreover the velocity for an Oldroyd-B fluid is larger when compared with the Burgers' fluid. The increasing effects of the Reynolds number $R$ on $T$ are shown in Fig. 3a. Figure 3b depicts the decreasing effects of Burgers' fluid parameter $\beta$ on $T$. Figure $3 \mathrm{c}$ illustrates that critical value of $T$ decreases with an increase in 
Table 1

Variation of mean-velocity distribution and reversal flow $\bar{u}(y)$ for different fluids

\begin{tabular}{lc}
\hline Types of fluids & $m=0.01, B=2, T=1, K=1$, \\
& $R=10, d=0.2, \alpha=0.5, \epsilon=0.15$ \\
Viscous fluid & 0.0000679084 \\
Maxwell fluid & 0.0000224859 \\
Oldroyd-B fluid & 0.0000501488 \\
Burgers' fluid & 0.000035005 \\
\hline
\end{tabular}

$\lambda$. The effects of $\lambda_{r}$ (Fig. 3d) on critical value of $T$ are quite opposite to those of $\lambda$. We further note that the critical value of $T$ is very high for small values of the wave number $\alpha$ when compared with its large values. Fig. 3e represents the behavior of the spring stiffness coefficient $K$ on $T$. It is observed from this figure that $T$ decreases with increasing $K$. A similar effect is seen in Fig. $3 f$ which elucidates the effect of the flexural rigidity of the plate on $T$.

\section{Closing remarks}

The main theme of this article is to discuss the effect of wall compliance on the peristaltic flow of a Burgers' fluid. The relevant equations are modeled and the results are presented for the free pumping case. The main conclusions are given below

- The behaviors of $\lambda$ and $\lambda_{r}$ on the mean velocity are different.

- The constant $D$ decreases with an increase in $d$, $\lambda$ and $\beta$.

- The value of $D$ increases when $T$ and $\lambda_{r}$ are increased.

- $T$ decreases when $\beta$ is increased.

- The flow reversal increases by increasing $\lambda_{r}$ and $T$. However it decreases when $d, \lambda$ and $\beta$ are increased.

- The corresponding results can be deduced for viscous fluid [17] when $\beta=\lambda=\lambda_{r}=0$, Maxwell fluid for $\beta=\lambda_{r}=0$ and an Oldroyd-B fluid for $\beta=0$.

\section{References}

[1] M.Y. Jaffrin and A. Shapiro, Peristaltic pumping, Annual Review of Fluid Mechs 3 (1971), 13-16.

[2] M.H. Haroun, Effect of relaxation and retardation time on peristaltic transport of the Oldroydian viscoelastic fluid, $J$ Appl Mech and Technical Phys 46 (2005), 842-850.
[3] Kh.S. Mekheimer and Y. Abd Elmaboud, Peristaltic flow of a couple stress fluid in an annulus: Application of an endoscope, Physica A 387 (2008), 2403-2415.

[4] Kh.S. Mekheimer and Y. Abd Elmaboud, Peristaltic flow through a porous medium in an annulus: application of an endoscope, Appl Math Info Sci 2 (2008), 103121.

[5] T. Hayat, Z. Asghar, S. Asghar and S. Mesloub, In fluence of inclined magnetic field on peristaltic transport of fourth grade.uid in an inclined asymmetric channel, J Taiwan Institute of Chem Eng 41 (2010), 553-563.

[6] Y. Abd Elmaboud and Kh.S. Mekheimer, Non-linear peristaltic transport of a second-order fluid through a porous medium, Appl Math Model 35 (2011), 26952710.

[7] S. Srinivas and R. Muthuraj, Effects of chemical reaction and space porosity on MHD mixed convective flow in a vertical asymmetric channel with peristalsis, Math Comp Model 54 (2011), 1213-1227.

[8] S. Srinivas, R. Gayathri and M. Kothandapani, Mixed convective heat and mass transfer in an asymmetric channel with peristalsis, Comm Nonlinear Sci Numer Simul 16 (2011), 1845-1862.

[9] T. Hayat, H. Yasmin and M. Al Yami, Soret and Dufour effects in peristaltic transport of physiological.uids with chemical reaction: A mathematical analysis, Computers and Fluids 89 (2014), 242-253.

[10] Y. Abd Elmaboud, In fluence of induced magnetic field on peristaltic flow in an annulus, Comm Nonlinear Sci Numer Simul 17 (2012), 685-698.

[11] S. Srinivas, A.S. Reddy and T.R. Ramamohan, A study on thermal-diffusion and diffusion-thermo effects in a twodimensional viscous flow between slowly expanding or contracting walls with weak permeability, Int J Heat Mass Transfer 55 (2012), 3008-3020.

[12] D. Tripathi and O.A. Beg, A study of unsteady physiological magneto-fluid flow and heat transfer through a finite length channel by peristaltic pumping, Proceedings of the Institution of Mechanical Engineers, Part H, Journal of Engineering in Medicine 226 (2012), 631-644.

[13] D. Tripathi, A mathematical model for swallowing of food bolus through the oesophagus under the in fluence of heat transfer, International Journal of Thermal Sciences $\mathbf{5 1}$ (2012), 91-101.

[14] N.S. Akbar, T. Hayat, S. Nadeem and S. Obaidat, Peristaltic flow of a Tangent hyper-bolic.uid in an inclined asymmetric channel with slip and heat transfer, Prog Comp Fluid Dynamics 12 (2012), 363-374.

[15] D. Tripathi, Study of transient peristaltic heat flow through a finite porous channel, Math Comp Model 57 (2013), 1270-1283.

[16] D. Tripathi and O. Anwar Beg, Transient magneto-peristaltic flow of couple stress biofluids: A magneto-hydro-dynamical study on digestive transport phenomena, Mathematical Biosciences In press 246 (2013), 72-83.

[17] M.A. Abd Elnaby and M.H. Haroun, A new model for study the effect of wall properties on peristaltic transport of a viscous fluid, Comm Nonlinear Sci Numer Simul 13 (2008), $752-762$.

[18] Kh.S. Mekheimer and A.N. Abdel Wahab, Effect of wall compliance on compressible fluid transport induced by a surface acoustic wave in a microchannel, Num Methods for Partial Diff Eqs 27 (2011), 621-636. 
[19] M. Mustafa, S. Hina, T. Hayat and A. Alsaedi, In fluence of wall properties on the peristaltic flow of a nano fluid: Analytic and numerical solutions, Int J Heat and Mass Trans 55 (2012), 4871-4877.

[20] T. Hayat, M. Javed and A.A. Hendi, Wall compliance effect on the flow of compressible non-Newtonian fluid, $J$ Mech Med Bio 12 (2012), 1250004-1250017.
[21] A.V. Ramana Kumari and G. Radhakrishnamacharya, Effect of slip and magnetic field on peristaltic flow in an inclined channel with wall effects, Int J Biomath 5 (2012), 1250015-1250031.

[22] Y.C. Fung and C.S. Yih, Peristaltic transport, ASME J Appl Mech 33 (1968), 669-675. 



Submit your manuscripts at

http://www.hindawi.com
